\title{
The processing of asymmetric and symmetric sentential conjunction
}

Ellen Thompson, Javier Collado, Maria Omana, Amanda Yousuf-Little Linguistics Program, Department of English, Florida International University, U.S.A.

https://doi.org/10.36505/ExLing-2011/04/0032/000201

\begin{abstract}
In this study, we examine the predictions for processing of a syntactically articulated theory of the distinction among different interpretations of clausal 'and'. Bjorkman (2010) claims that symmetric 'and' interpretations involve coordination of CPs; these are logical interpretations. Asymmetric interpretations of 'and' involve conjunction of TPs; these are temporal and causal. If the processor is guided by structural considerations, we predict a possible two-way split in the processing costs of these structures. Therefore, this research examines the processing time involved in sentences interpreted as: (i) temporal, (ii) causal, and (iii) logical, versus the distinctions of (i) asymmetric (TP structure), and (ii) symmetric (CP structure). We find that structures involving symmetric 'and' involve longer processing times than those of asymmetric, causal 'and', and although the processing times of structures with logical 'and' are longer than those with temporal 'and', this distinction does not approach statistical significance.
\end{abstract}

Key words: coordination, conjunction, processing time

\section{Introduction}

Within Generative Grammar, multiple analyses of the distinct interpretations associated with the clausal conjunct 'and' have been developed (Culicover 1970; Posner 1980). This paper focuses on the logical interpretation of 'and' illustrated in (1a), the temporal interpretation shown in (1b), and the causal meaning, as in (1c):

(1) a. Water freezes at $0 \circ \mathrm{C}$ and ethanol freezes at $-114 \circ \mathrm{C}$.

b. The lights came on and the singer stepped onto the stage.

c. The lights were off and I couldn't see.

It has been noted that logical 'and' is symmetric; it allows a reversal of the two conjuncts with a maintenance of meaning; (2a) is equivalent to (1a):

(2) a. Ethanol freezes at $-114 \circ \mathrm{C}$ and water freezes at $0 \circ \mathrm{C}$.

In contrast, temporal and causal 'and' do not permit reversal of the two conjuncts with the same meaning, ((3a) versus (1b) and (3b) versus (1c)) and these uses are therefore characterized as asymmetric.

ExLing 2011: Proceedings of 4th Tutorial and Research Workshop on Experimental Linguistics, 25-27 May, Paris, France 
(3) a. The singer stepped onto the stage and the lights came on.

b. I couldn't see and the lights were off.

In contrast to earlier claims that it is pragmatic and discourse factors which determine the interpretation of clausal 'and', Bjorkman (2010) argues that the difference between symmetric and asymmetric 'and' is semantic. She claims that this semantic distinction is reflected in the syntax of the conjunction structures: symmetric coordination involves conjunction of $\mathrm{CP}$ structures, whereas asymmetric coordination involves conjunction of TP structures.

We investigate the prediction for processing of such a syntactically articulated theory. It is predicted by this approach that the processing cost associated with the comprehension and production of these two interpretations of clausal 'and' is different. Processing of asymmetric conjunction structures involve conjunction of TPs, and thus less structure than the processing of symmetric 'and' structures, which require the conjunction of necessarily larger structures, CPs. Assuming that processing cost is associated with syntactic structure that is phonologically covert, as well as with phonologically overt material, the difference in processing time between these two distinct types of coordination is predicted to be measurable.

\section{Methods}

We tested this hypothesis with eight adult monolingual English speakers using the Rapid Serial Visual Presentation (RSVP) method via a PowerPoint presentation. Subjects are displayed sixty sentences in total, composed of thirty filler sentences as well as ten causal, ten temporal, and ten logical sentences.

Sentences are displayed a single word at a time for $800 \mathrm{~ms}$. Participants silently repeat each word to themselves, without labial movement. At the end of each sentence, participants are prompted by a marker " $X$ " and a tone. This indicates the completion of the sentence and prompts the subject to reproduce the target sentence. The processing time of each coordinate structure is processed using WavePad Sound Editor by measuring the distance between the beginning of the sounded prompt and the completion of the participants' utterance.

\section{Results}

A one way repeated measure ANOVA was carried out on the processing times for the three coordinate structures. Post hoc tests were carried out using Fisher's LSD pair wise comparison at the 5\% significance level. There was a significant difference in coordinate structures, $\mathrm{F}(2,14)=6.86, \mathrm{p}<.017$. Fisher's tests indicated that the mean processing time for causal $(\mathrm{M}=3.48$ 
The processing of asymmetric and symmetric sentential conjunction 133

sec) was significantly less than temporal $(\mathrm{M}=3.86)$ and logical $(\mathrm{M}=4.23)$. A significant difference was not found between temporal and logical and structures. Refer to Table 1, Comparison of Production Times of Coordinate Structures.

Table 1. Comparison of Production Times of Coordinate Structures

\begin{tabular}{|l|r|r|}
\hline & \multicolumn{1}{|c|}{$\begin{array}{c}\text { Asymp. } \\
\text { Sig. } \\
\text { (2-tailed) }\end{array}$} \\
\hline temporal - causal & \multicolumn{1}{|c|}{$-1.960^{\mathrm{a}}$} & .050 \\
logical - causal & $-2.100^{\mathrm{a}}$ & .036 \\
logical - temporal & $-1.540^{\mathrm{a}}$ & .123 \\
\hline
\end{tabular}

Due to the presence of several high processing times, the nonparametric Friedman's test was performed to confirm the ANOVA results; similar results were found. There was a significant difference among the three coordinate structures, $\mathrm{X} 2(2, \mathrm{~N}=8)=7.00, \mathrm{p}<.03$. The causal structure had significantly less processing time than the temporal and logical structures, while temporal and logical did not differ between them.

Figure 1 illustrates the mean production time of each coordinate structure, demonstrating the higher processing time associated with logical and temporal conjunctions in contrast to causal and conjunctions.

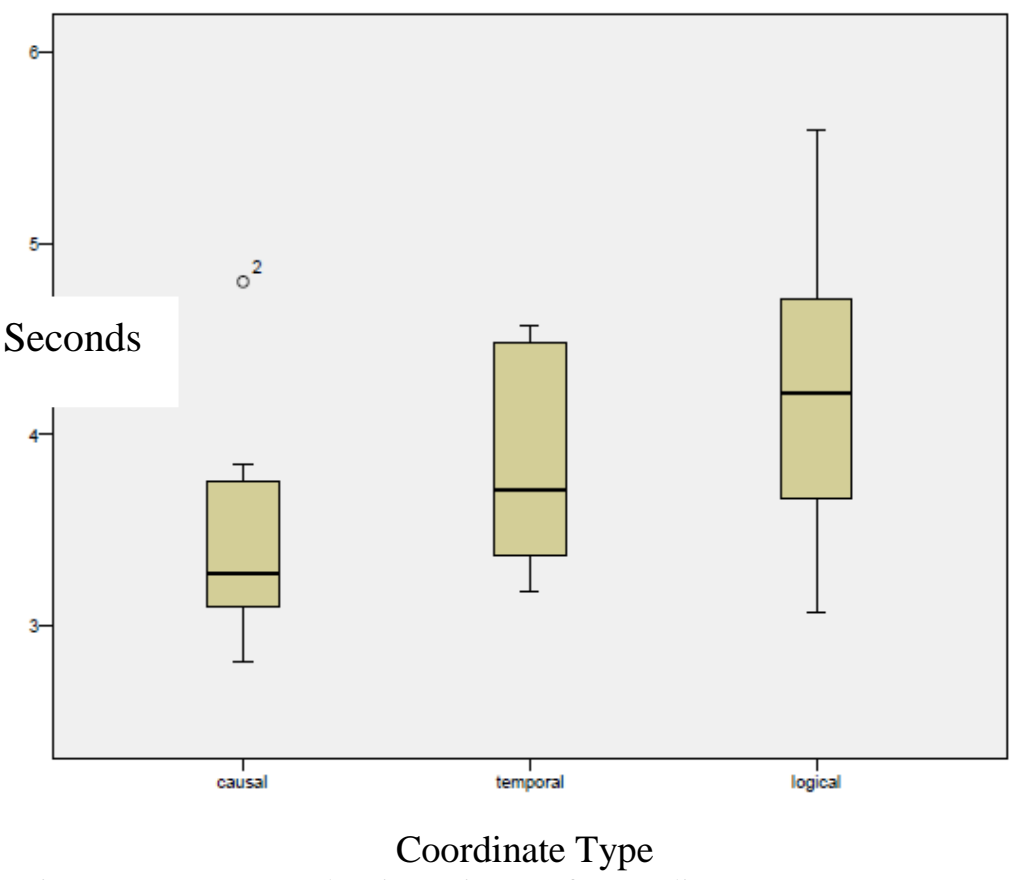

Figure 1. Mean Production Times of Coordinate Structures 


\section{Discussion}

Recall that the prediction of a two-way distinction between the syntactic and semantic structures of symmetric and asymmetric 'and' is that logical 'and' structures should have a higher processing time than causal and temporal 'and' structures. The data from this study provide partial confirmation of these predictions: structures involving symmetric 'and' involve longer processing times than those of asymmetric, causal 'and'. However, although it is the case that the processing times of structures with logical 'and' are longer than those with temporal 'and', this distinction does not approach statistical significance. This effect is not predicted, and the implications of this effect for the theory of coordination are to be discussed.

\section{Acknowledgements}

We would like to extend our gratitude for financial support for this research to the Graduate Student Funding Committee, the College of Arts and Sciences, and the English Department at Florida International University. We also want to thank for their support and comments: Antonis Botinis, Gisela Casines, Marcela Depiante, Maureen Donnelly, Ana Luszczynska, James Sutton, and Kezia Walker.

\section{References}

Blakemore, D., Carston, R.. 2005. The pragmatics of sentential coordination with 'and'. Lingua 115.

Bjokman, B. 2010. A Syntactic Correlate of a Semantic Asymmetry, paper presented at NELS 41, October.

Culicover, P.W. 1970. One more can of beer. Linguistic Inquiry 1, 366-369.

Culicover, P.W., R.S. Jackendoff. 1997. Semantic subordination despite syntactic coordination. Linguistic Inquiry 28, 195-217.

Posner, R. 1980. Semantics and pragmatics of sentence connectives in natural language. Speech act theory and pragmatics 168-203.

Progovac, L. 1998. Structure for coordination. Part II. Glot International 3,3-9.

Schmerling, S.F. 1975. Asymmetric conjunction and rules of conversation. In Cole, P., Morgan, J.L. (eds.), Syntax and semantics volume 3, Speech Acts, 211-232. Academic Press.

Txurruka, I.G. 2003. The natural language conjunction 'and'. Linguistics and Philosophy 26:255-285. 